\title{
Distinct effects of Lactobacillus plantarum KL30B and Escherichia coli 3A1 on the induction and development of acute and chronic inflammation
}

\author{
MAGDALENA STRUS ${ }^{1,2}$, KRZYSZTOF OKON ${ }^{3}$, BERNADETA NOWAK ${ }^{4}$, \\ MAGDALENA PILARCZYK-ZUREK', PIOTR HECZKO ${ }^{l}$, ANNA GAWDA ${ }^{4}$, MARTA CISZEK-LENDA ${ }^{4}$, \\ BEATA SKOWRON ${ }^{5}$, AGNIESZKA BARANOWSKA ${ }^{5}$, JANUSZ MARCINKIEWICZ2,4 \\ ${ }^{1}$ Chair of Microbiology, Jagiellonian University Medical College, Krakow, Poland \\ ${ }^{2}$ University Center for Veterinary Medicine, Jagiellonian University and University of Agriculture, Krakow, Poland \\ ${ }^{3}$ Chair of Pathomorphology, Jagiellonian University Medical College, Krakow, Poland \\ ${ }^{4}$ Chair of Immunology, Jagiellonian University Medical College, Krakow, Poland \\ ${ }^{5}$ Department of Pathophysiology, Jagiellonian University Medical College, Krakow, Poland
}

\begin{abstract}
Objective: Enteric bacteria are involved in the pathogenesis of ulcerative colitis. In experimental colitis, a breakdown of the intestinal epithelial barrier results in inflow of various gut bacteria, induction of acute inflammation and finally, progression to chronic colitis.

Material and methods: In the present study we compared pro-inflammatory properties of two bacterial strains isolated from human microbiome, Escherichia coli 3A1 and Lactobacillus plantarum KL3OB. The study was performed using two experimental models of acute inflammation: peritonitis in mice and trinitrobenzenesulfonic acid (TNBS)-induced colitis in rats.

Results: Both bacterial strains induced massive neutrophil infiltration upon injection into sterile peritoneal cavity. However, peritoneal exudate cells stimulated in vitro with E. coli 3A1, produced far more nitric oxide, than those stimulated with L. plantarum KL3OB. Interestingly, distinct effect on the development of TNBS-induced colitis was observed after oral administration of the tested bacteria. Lactobacillus plantarum KL3OB evoked strong acute colitis. On the contrary, the administration of E. coli $3 A 1$ resulted in a progression of colitis to chronicity.

Conclusions: Our results show that distinct effects of bacterial administration on the development of ongoing inflammation is strain specific and depends on the final effect of cross-talk between bacteria and cells of the innate immune system.
\end{abstract}

Key words: TNBS-induced colitis, Escherichia coli, Lactobacillus plantarum, inflammation, neutrophils.

(Cent Eur J Immunol 2015; 40 (4): 420-430)

\section{Introduction}

Ulcerative colitis (UC), as well as Crohn's disease, belong to inflammatory bowel disease (IBD), chronic and spontaneously relapsing inflammatory disorders of unknown aetiology. Although the pathogenesis of IBD is still not fully understood, there is an increasing number of experimental data suggesting the role of various gut bacteria in the development (propagation/perpetuation) of chronic inflammation in IBD [1-3].

Among them, special attention has been paid to a possible role of Escherichia coli subtypes in pathogenesis of
IBD, especially UC, as E. coli are the predominant aerobic Gram-negative bacteria of the gut microbiome, and an increased number of $E$. coli in inflamed tissue in the gut of UC patients have been demonstrated [4]. Moreover, apart from various commensal strains, some diarrhoeagenic pathotypes of $E$. coli have been identified (e.g. shiga toxin-producing E. coli) [5]. They possess virulent properties such as adherence to the gut mucosa, production of enterotoxins and tissue invasion [6]. These pathogenic E. coli strains can cause acute diarrhoea, but with no documented role in the pathogenesis of IBD. So far there is no proof

Correspondence: Janusz Marcinkiewicz, Department of Immunology, Jagiellonian University Medical College, Czysta 18, 31-121 Krakow, Poland, e-mail: mmmarcin@cyf-kr.edu.pl 
that any particular E. coli strain can cause ulcerative colitis. However, some pathogenic E. coli strains with pro-inflammatory properties may play a role in the exacerbation of UC $[7,8]$.

On the other hand, it is tempting to speculate that the increased number of non-pathogenic E. coli in inflamed tissue may have beneficial effect and may be involved in an amelioration and resolution of intestinal inflammation. Only recently, we have reported that E. coli strains isolated from patients with UC attenuate the detrimental Fenton's reaction by inhibiting the formation of hydroxyl radicals, important toxic agents of chronic inflammation [7]. It points to anti-inflammatory properties of these E. coli strains. Moreover, it has been demonstrated in clinical trials that non-pathogenic E. coli strain Nissle 1917 was as effective as 5-ASA (5-aminosalicylic acid: the primary anti-inflammatory drug used to treat IBD) in preventing relapse and maintenance of remission of ulcerative colitis [9]. All these data confirm dual role of $E$. coli in pathogenesis in UC and its probiotic potential. Indeed, E. coli strain Nissle 17 bacteria, along with various Lactobacillus strains (L. plantarum, L. reuterii, L. fermentum) are the most common probiotics used in the treatment of inflammatory bowel disease including UC [10-12].

The majority of data showing the preventive effect of probiotic bacteria on the development of UC was demonstrated in animal models of acute and chronic colitis [1316]. However, further studies are necessary to explain in details their beneficial role in the regulation of acute and chronic inflammation and to estimate anti-inflammatory profile of the tested bacterial strains, as immunoregulatory properties of bacteria are strain-specific. The required anti-inflammatory properties of probiotics are revealed by catalase positive bacterial strains, such as Lactobacillus plantarum KL30B [3].

The aims of the present study were: 1) to compare the effect of administration of the selected strain of $E$. coli (3A1) and L. plantarum (KL30B) on the induction of acute peritonitis in mice; 2) to compare the effect of these bacteria on the development of TNBS-induced colitis in rats; 3 ) to compare their ability to stimulate the production of inflammatory mediators by in vitro activated murine peritoneal macrophages.

\section{Material and methods}

\section{Mice}

CBA/J male mice (8-12 weeks of age, 18-22 g) were maintained in the Animal Breeding Unit, Department of Immunology, Jagiellonian University Medical College. All mice were held in standard caging conditions with water and standard diet ad libitum. The authors were granted permission by the Local Ethics Committee to use mice in this study.

\section{Rats}

Wistar male rats (12-14 weeks of age, weighing approx. $300 \mathrm{~g}$ ), were purchased from Animal Breeding Unit at the Faculty of Pharmacy Jagiellonian University Medical College and housed in the Animal Unit of Pathophysiology Department (Jagiellonian University Medical College). Animals were held in standard caging conditions with ad libitum access to water and standard rodent food (Labofeed, Kcynia, Poland). The use of rats in this study and all experimental procedures were approved by the Local Ethics Committee.

\section{Bacterial strains \\ Characterization and preparation of Escherichia coli strain}

Escherichia coli $3 \mathrm{~A} 1$ strain used in the experiments was selected from over 100 strains isolated from the colonic mucosa with inflammatory changes of patients with a diagnosis of Crohn's disease. The strain was selected basing on frequency analyses of the genes responsible for synthesis of proteins for acquisition of iron ions. The selected strain had the following genes encoding: $f e o B$ - high affinity ferrous iron transporter, $c h u A$ - heme uptake protein, fepA, iroN, iutA, fhuA, fecA, fyu - receptor proteins for siderophores: enterobactin, salmochelin, aerobactin, ferrichrome, rhizoferrin, yersiniabactin, respectively. Additionally, the selected strain of E. coli $3 \mathrm{~A} 1$ produces two types of hydroperoxidases: catalase/peroxidase I (HPI) and hydroperoxidase II (HPII), encoded by kat $G$ and katE, respectively. The strain showed the highest level of catalase biosynthesis following the induction with iron.

For in vivo studies both living and killed E. coli $3 \mathrm{~A} 1$ bacteria were used. For this purpose, the selected E. coli $3 \mathrm{~A} 1$ strain was grown overnight at $37^{\circ} \mathrm{C}$ in MacConkey agar (Oxoid, Thermo Scientific, UK). This strain was inoculated in $10 \mathrm{ml}$ of tryptic soy broth (TSB) (BD, USA) and incubated at $37^{\circ} \mathrm{C}$ for $3 \mathrm{~h}$. After incubation, bacterial culture was microcentrifuged $\left(5000 \mathrm{rpm}, 10 \mathrm{~min}, 4^{\circ} \mathrm{C}\right)$, the supernatant was removed, and pellet was resuspended in $10 \mathrm{ml}$ of phosphate buffered saline (PBS). This step was repeated three times. Subsequently, the sample was resuspended in $5 \mathrm{ml}$ PBS. Bacterial density in the sample was determined by measuring optical density (JASCO Corporation Spectra Manager v.1.30.01) at a wavelength of 600 $\mathrm{nm}\left(\mathrm{OD}_{600}\right)$. Inocula of the E. coli $3 \mathrm{~A} 1$ bacteria had the OD reading $2.5 \pm 0.02$ which corresponds to $5 \times 10^{8}$ colony forming units $(\mathrm{CFU}) / \mathrm{ml}$.

Bacterial cells were heat-killed by sterilization at $120^{\circ} \mathrm{C}$ for $20 \mathrm{~min}$.

\section{Characterization and preparation of \\ Lactobacillus plantarum strain}

Lactobacillus plantarum KL30B strain was selected from the gastrointestinal tract of a healthy human adult. It had unique antioxidant properties thanks to the production 
of catalase and liberation it outside of the bacterial cells. The chemical decomposition of hydrogen peroxide to oxygen and water by fresh culture of L. plantarum KL30B was detected by colorimetric OxiSelect Hydrogen Peroxide Assay Kit (Cell Biolabs, USA).

The fresh culture of L. plantarum KL30B was prepared in $10 \mathrm{ml}$ of MRS agar broth (Oxoid, Thermo Scientific, $\mathrm{UK})$ at $37^{\circ} \mathrm{C}$ in anaerobic condition. After $48 \mathrm{~h}$ of incubation, the culture was centrifuged ( $3000 \mathrm{rpm}, 15 \mathrm{~min}, 4^{\circ} \mathrm{C}$ ). The pellet of L. plantarum KL30B was suspended in $1 \mathrm{ml}$ of PBS (this step was repeated twice). The final density of live cultures of L. plantarum KL30B for oral administration to rats, was set at 0.5 MacFarland scale, which corresponded to $1 \times 10^{9} \mathrm{CFU} / \mathrm{ml}$ of PBS.

\section{Experimental models}

\section{In vitro activation of murine macrophages}

Peritoneal mouse macrophages (PEC) were induced by intraperitoneal injection of $3 \mathrm{ml}$ of thioglycollate (3-4 mice). Four days later, cells accumulated in peritoneal cavity were harvested (washed out with PBS containing $1 \%$ heparin). After washing the cells 3 times in IMDM containing 5\% FBS, $25 \mathrm{mM}$ HEPES, $2 \mathrm{mM}$ L-glutamine and $0.05 \mathrm{mg} / \mathrm{ml}$ gentamycin, they were seeded in $24-$ well plates at the density of $5 \times 10^{5} /$ well. Nonadherent cells were washed out 2-3 h later. Adherent cells (macrophages) were cultured in the presence of LPS $(100 \mathrm{ng} / \mathrm{ml})$ or bacteria $\left(100 \mu \mathrm{l}\right.$ of $1 \times 10^{8} \mathrm{CFU} / \mathrm{ml}$ per well) either E. coli $3 \mathrm{~A} 1$ (killed) or L. plantarum KL30B (live) or both E. coli 3A1 (killed) and L. plantarum KL30B (live) $\left(100 \mu \mathrm{l}\right.$ of $1 \times 10^{8}$ $\mathrm{CFU} / \mathrm{ml}$ each per well). Culture supernatants were collected $24 \mathrm{~h}$ later and saved for cytokine ELISA and NO assay.

\section{Induction of acute peritonitis in mice}

Acute peritonitis was induced by intraperitoneal injection of bacteria $\left(100 \mu \mathrm{l} /\right.$ mouse of $\left.1 \times 10^{8} \mathrm{CFU} / \mathrm{ml}\right)$ either L. plantarum KL30B (live or killed), killed E. coli $3 \mathrm{~A} 1$ or a mixture of live L. plantarum KL30B and killed E. coli $3 \mathrm{~A} 1\left(100 \mu \mathrm{l} / \mathrm{mouse}\right.$ of $1 \times 10^{8} \mathrm{CFU} / \mathrm{ml}$ each). One group of mice received LPS $(1 \mu \mathrm{g} /$ mouse in $100 \mu \mathrm{l})$. Naïve untreated mice were used as a control. Each experimental group consisted of 5-6 mice. Eighteen hours later, peritoneal exudate cells were collected by washing out the peritoneal cavity with $5 \mathrm{ml}$ of PBS containing $5 \mathrm{U} / \mathrm{ml}$ heparin (Polfa, Warsaw, Poland). Cells were centrifuged and red blood cells were lysed by osmotic shock with sterile distilled water. Equal volume of $2 \times$ concentrated PBS was used immediately to restore osmolality. After washing, the cells were used for myeloperoxidase (MPO) assay and stained for the flow cytometry to assess the percentage of neutrophils.

\section{TNBS-induced colitis in rats}

Rats were randomly divided into five groups $(n=5-8$ rats/group). Colitis was induced in all experimental groups
(1-5) by rectal administration of 2,4,6-trinitrobenzenesulfonic acid (TNBS) solution in $30 \%$ ethanol (Sigma-Aldrich, USA) (35 mg/rat) on day 0 and day 5 . In addition, rats (groups 2-5) were orally administered with the tested bacterial strains $\left(100 \mu \mathrm{l} / \mathrm{rat}\right.$ of $\left.1 \times 10^{9} \mathrm{CFU} / \mathrm{ml}\right), 3$ times a day, for 11 consecutive days (day 2-12). Namely: group 2 received live E. coli $3 \mathrm{~A} 1$, group 3 - killed E. coli $3 \mathrm{~A} 1$, group 4 - live L. plantarum KL30B. Rats in group 5 received live L. plantarum KL30B on day 2, killed E. coli 3A1 on day 3 and then for consecutive 9 days (day 4-12) living L. plantarum KL30B together with killed E. coli $3 \mathrm{~A} 1$. On day 12 (at the end of the experiment), rats were sacrificed by intraperitoneal administration of $100 \mathrm{mg} / \mathrm{kg}$ pentobarbital (Morbital, Biowet Pulawy, Poland). The rat colons were collected for further examination.

\section{Histological evaluation of colitis}

The selected fragments of intestine were fixed in $10 \%$ buffered formalin and sliced with a sharp blade into $3 \mathrm{~mm}$ long segments. These were routinely processed and paraffin embedded. Then, $2 \mu \mathrm{m}$ thick sections were cut out from the paraffin blocks and stained with haematoxylin and eo$\sin$. The slides were assessed (by microscopic observation) and classified as acute or chronic lesions. The acute lesions were defined basing on the presence of mucosal infiltration by polymorphonuclears (neutrophils). Chronic lesions were defined basing on the presence of mononuclear inflammatory infiltrates (composed mainly of lymphocytes and macrophages) and granulation tissue. Ulcerations were classified as acute or chronic lesions depending on the type of infiltrate and presence of healing (granulation tissue).

In addition, we applied the following histological grading scoring system to classify intestinal inflammation (Score 0-3):

- 0 - No inflammation,

- 1 - Slight infiltration of cells in lamina propria (mild acute inflammation),

- 2 - Massive infiltration of neutrophils (moderate acute inflammatory lesion),

- 3 - Ulceration and a presence of granulation tissue (chronic type lesion).

\section{Determination of nitric oxide production}

The level of nitric oxide (NO) in the culture supernatant of peritoneal thioglycollate-induced macrophages was measured by the accumulation of nitrite as a stable end product according to a modified Griess method [17]. Cell culture supernatant $(100 \mu \mathrm{l})$ was mixed with $14 \mathrm{mM}$ 4,4'-diamino-diphenylsulphone (Dapsone; Sigma Aldrich, USA) in $2 \mathrm{M} \mathrm{HCl}(50 \mu \mathrm{l})$ and $0.1 \% \mathrm{~N}$-1-naphtylenediamine dihydrochloride ( $50 \mu \mathrm{l})$ (POCH Gliwice, Poland). The absorbance of tested culture supernatants measured at $550 \mathrm{~nm}$ was compared with a sodium nitrate standard $\left(\mathrm{NaNO}_{2}\right)$ curve. 


\section{Measurement of myeloperoxidase enzymatic activity}

Myeloperoxidase activity was measured according to the Bradley's method [18] with slight modifications. Shortly, intestinal tissue pieces (TNBS-induced colitis in rats) or cells collected from peritoneal cavity $18 \mathrm{~h}$ after injection of LPS or bacteria (acute peritonitis in mice) were placed in a $50 \mathrm{mM}$ phosphate buffer, $\mathrm{pH} 6.0$, containing $0.5 \%$ HTAB (hexadecyltrimethyl-ammonium bromide) (Sigma Aldrich, USA) and disrupted by three cycles of freezing and thawing. Tissue samples were additionally homogenized using Ultrasonic Homogenizer Sonopuls (Bandelin electronic GmbH, Germany). After centrifugation (4000 g, $15 \mathrm{~min}$ ) supernatants were collected. Aliquots of tested supernatants $(0.1 \mathrm{ml})$ were mixed with $2.9 \mathrm{ml}$ phosphate buffer $(50 \mathrm{mM})$ containing o-dianisidine dihydrochloride $(0.167 \mathrm{mg} / \mathrm{ml})$ and hydrogen peroxide $(0.0005 \%)$ and incubated $20 \mathrm{~min}$ at room temperature. The absorbance was measured at $460 \mathrm{~nm}$. Recombinant MPO (Sigma Aldrich, USA) was used as a standard. The activity of MPO was expressed in units. One unit of MPO activity was defined as that degrading $1 \mu \mathrm{mol}$ of $\mathrm{H}_{2} \mathrm{O}_{2}$ per minute at room temperature. The protein level in the tissues samples (intestine) was measured using Bicinchoninic Acid Protein Assay Kit (Sigma Aldrich, USA) and the MPO activity was expressed in units per mg protein.

\section{Determination of cytokines by ELISA}

Cytokines in cell culture supernatant were measured by sandwich ELISA. Microtiter plates (Costar EIA/RIA plates, Corning Incorporated, USA) were coated with cytokine specific antibody. Interleukin (IL)-10 and tumor necrosis factor $\alpha$ (TNF- $\alpha$ ) were measured according to the manufacturer's instruction of OptEIA mouse IL-10 Set (BD Biosciences, USA) and mouse TNF- $\alpha$ ELISA Ready-Set-Go (eBioscience, USA). For IL-6 assay plates were coated with monoclonal rat anti-IL-6 antibodies (BD Biosciences, USA) followed by blocking with $3 \%$ low fat milk (IL-6). Recombinant IL-6 (eBioscience, USA) and cell culture supernatant was added into the plates followed by biotinylated rat anti-mouse IL-6 antibody (eBioscience, USA), and streptavidin-HRP (Vector Laboratories, USA). Hydrogen peroxide (Sigma Aldrich, USA) in the presence of chromogenic dye TMB (BioLegend, USA) was used to develop colorimetric reaction. The reaction was stopped with $2 \mathrm{M}$ sulfuric acid (POCH Gliwice, Poland) and the optical density was measured at 450 (570) nm using the microtiter plate reader (PowerWaveX, Bio-Tek Instruments, Winooski, VT).

\section{Flow cytometry}

Peritoneal exudate cells collected $18 \mathrm{~h}$ after injection of LPS or bacteria were washed with PBS containing 2\% FBS and $0.05 \%$ sodium azide. Cells were stained with bio- tin conjugated anti-mouse Gr1 (Ly-6G/Ly-6C) monoclonal antibody (BioLegend, USA) followed by APC-Streptavidin (BD Biosciences). Cells were co-stained with PE-conjugated rat anti-mouse F4/80 monoclonal antibody (eBioscience, USA). Propidium iodide (PI) (BD Bioscences, USA) was added to the cells just before the analysis to exclude dead cells. Cells were analyzed in FACS Calibur (BD Biosciences) with CellQuestPro Sotware. Live (PI negative), FCS $^{\text {hi }}$ cells were gated and analyzed for the expression of Gr1 (as a marker of neutrophils) and F4/80 (monocyte/macrophage marker).

\section{Statistical analysis}

Statistical significance of differences between groups was analysed using One-way ANOVA, followed, if significant, by a Tukey's test for post hoc comparison. Analyses were performed using Graphpad Prism v. 5.01 (GraphPad Software, Inc., USA). In some cases (experiments, as indicated), Student's $t$-test was used (Microsoft Excel), if appropriate. A p-value less than 0.05 were considered statistically significant.

\section{Results}

\section{Effect of $E$. coli 3A1 and L. plantarum KL30B on the induction of acute peritonitis in mice}

Escherichia coli 3A1 (killed) and L. plantarum KL30B (live or killed) were injected intraperitoneally to compare their pathogenicity and capability to induce local acute inflammation in a sterile tissue without concomitant participation of host microbiome bacteria. Mice were injected with killed E. coli bacteria only, and not treated with live E. coli, to avoid systemic infection and life-threatening severe peritonitis. As shown in Table 1, both tested bacterial strains induced a similar total number of infiltrating peritoneal exudate cells. LPS alone, as compared to killed $E$. coli A31, was a much weaker inducer of inflammatory cells. Moreover, phenotypic analysis of the cells present in peritoneal cavity $18 \mathrm{~h}$ after the injection of microbial agents showed the high content of neutrophils in all experimental groups, as measured by a percentage of $\mathrm{Gr} 1^{+} \mathrm{F} 4 / 80^{-}$cells. The results were correlated with MPO activity of the tested cells (Tables 1,2). The highest percentage of neutrophils was observed in mice treated with killed $E$. coli $3 \mathrm{~A} 1$.

\section{Effect of $E$. coli 3A1 and L. plantarum KL30B on the production of inflammatory mediators by in vitro stimulated murine peritoneal exudate cells}

Unstimulated peritoneal exudate cells spontaneously produce negligible amounts of TNF- $\alpha$ and IL-6, and undetectable amounts of IL-10 and NO [19]. However, incubation of these cells in vitro with the tested microbial agents 
Table 1. Cells infiltrating peritoneal cavity and MPO activity in acute peritonitis induced by LPS or tested bacterial strains

\begin{tabular}{|c|c|c|}
\hline Group & MPO $\left[\mathrm{U} \times 10^{-3} / 10^{6} \text { cells }\right]^{\mathrm{b}}$ & The average cell number per mouse $\left[\times 1^{1} 0^{6}\right]^{\mathrm{a}}$ \\
\hline Control (naïve mice) & ND & $2.3 \pm 1.4$ \\
\hline LPS & $181 \pm 49$ & $1.4 \pm 1.3$ \\
\hline L. plantarum KL30B (live) & $194 \pm 37$ & $4.48 \pm 0.5$ \\
\hline L. plantarum KL30B (killed) & $287 \pm 68$ & $3.45 \pm 0.4$ \\
\hline E. coli $3 \mathrm{~A} 1$ (killed) & $321 \pm 44$ & $3.27 \pm 1.3$ \\
\hline $\begin{array}{l}\text { E. coli 3A1 (killed) } \\
+ \text { L. plantarum KL30B (live) }\end{array}$ & $277 \pm 30$ & $5.13 \pm 2.0$ \\
\hline
\end{tabular}

Table 2. The phenotype of cells infiltrating peritoneal cavity upon induction of acute peritonitis with LPS or tested bacterial strains

\begin{tabular}{lc}
\hline Group & Gr1+F4/80- cells [\%] \\
\hline Control (naïve mice) & $1.92 \pm 0.32$ \\
\hline LPS & $57.47 \pm 7.34$ \\
\hline L. plantarum KL30B (live) & $51.31 \pm 3.38$ \\
\hline L. plantarum KL30B (killed) & $60.16 \pm 0.87$ \\
\hline E. coli 3A1 (killed) & $73.02 \pm 5.30 *$ \\
\hline $\begin{array}{l}\text { E. coli 3A1 (killed) } \\
+ \text { L. plantarum KL30B (live) }\end{array}$
\end{tabular}

$\overline{\text { The percentage of cells expressing } \mathrm{Gr} 1 \text { (neutrophils) among cells infiltrating }}$ peritoneal cavity. Data shown are the mean $( \pm S D)$ of three independent experiments. ${ }^{*} p<0.05$ L. plantarum KL30B (killed) vs. E. coli $3 A 1$ (killed) (Student's $t$-test).

(LPS, whole bacteria) resulted in a massive release of both pro- (TNF- $\alpha$, IL-6, NO) and anti-inflammatory mediators (IL-10) (Fig. 1). All tested agents induced substantial secretion of IL- 6 with no differences between groups. On the contrary, important differences in the production of IL-10 and TNF- $\alpha$ were observed in particular groups. Namely, the cells activated with $L$. plantarum KL30B primarily released TNF- $\alpha$, while $E$. coli $3 \mathrm{~A} 1$ preferentially stimulated IL-10 formation. Importantly, E. coli $3 \mathrm{~A} 1$ shows strongest capacity to induce the production of NO. In some experiments the amount of NO released by the cells incubated with $E$. coli $3 \mathrm{~A} 1$ was even $>5 \times$ higher than the amount of NO induced by L. plantarum KL30B. In addition, the high- est production of NO was observed after the stimulation of the cells with both LPS and E. coli 3A1 (data not shown).

\section{Comparative effect of oral administration of E. coli 3A1 and L. plantarum KL30B on the development/progression of TNBS-induced colitis in rats}

Standard clinical evaluation of experimental chronic colitis in rats includes a control of body weight [20]. No weight loss was found in animals in our experimental model of colitis. Importantly, in contrast to mild clinical symptoms of colitis and lack of signs of anorexia, macroscopic and histological examinations of the colons showed common signs of inflammation such as hyperemia (Fig. 2), neutrophil infiltration and mucosa lesions (Fig. 3).

For the present study we have chosen the dose of TNBS which slightly damaged gut mucosa, after two administrations (see Material and methods), with the incidence of approximately $60 \%$. In these rats, colon inflammation involved few neutrophils in the mucosa and was classified as mild acute inflammation (histology score $=1$, $\mathrm{MPO}<100 \mathrm{U} \times 10^{-3} / \mathrm{mg}$ ).

As shown in Table 3, administration of either E. coli $3 \mathrm{~A} 1$, L. plantarum KL30B or both bacteria resulted in the development of more severe colitis than that observed in the TNBS group. The incidence of colitis classified by histological examination in all experimental groups was similar (60-71\%). In the E. coli 3A1 group, ulcerations with significantly lower level of MPO activity were present. Such lesions were classified as chronic inflammation (Fig. 4A). Lactobacillus plantarum KL30B administration was associated with a predominant neutrophil infiltration confirmed by a significantly higher level of MPO activity and the process was classified as acute inflammation (acute lesions) (Fig. 4B). In the group which received both bacteria, the observed lesions were characterised as acute ( 3 cases out of 7 ) or as a mixture of acute and chronic 
A

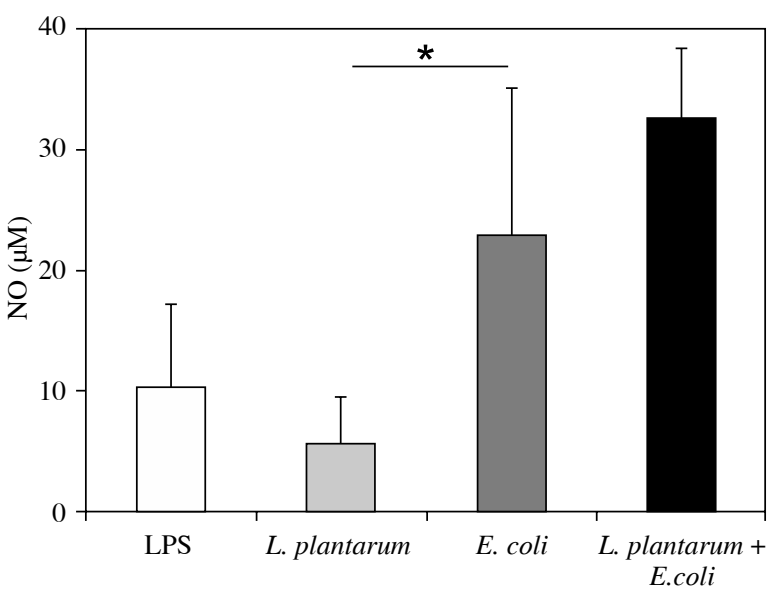

C

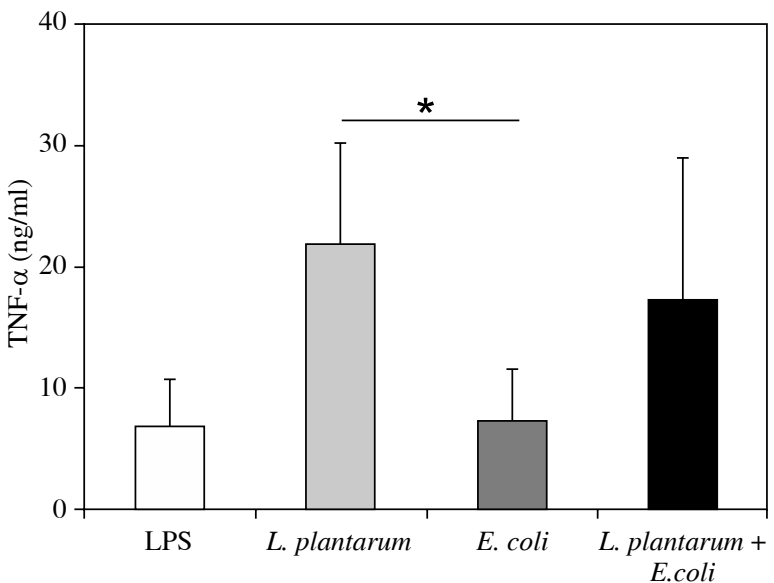

B

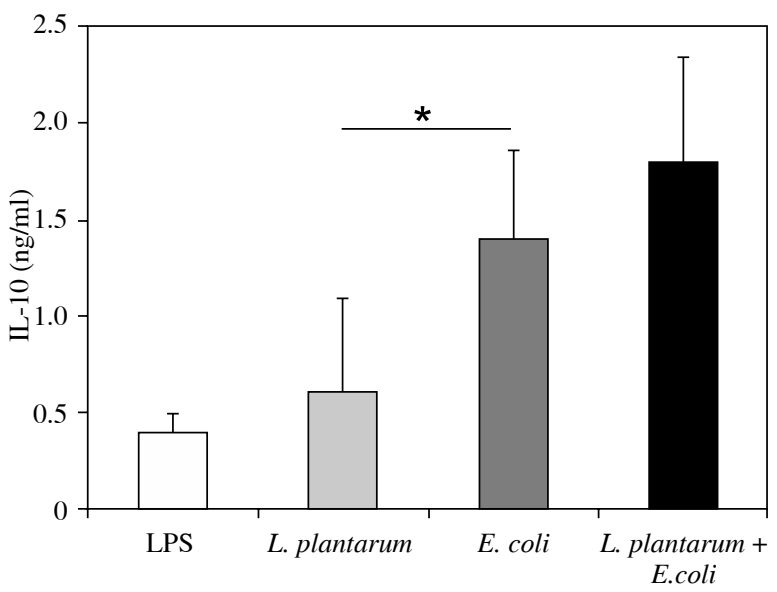

D

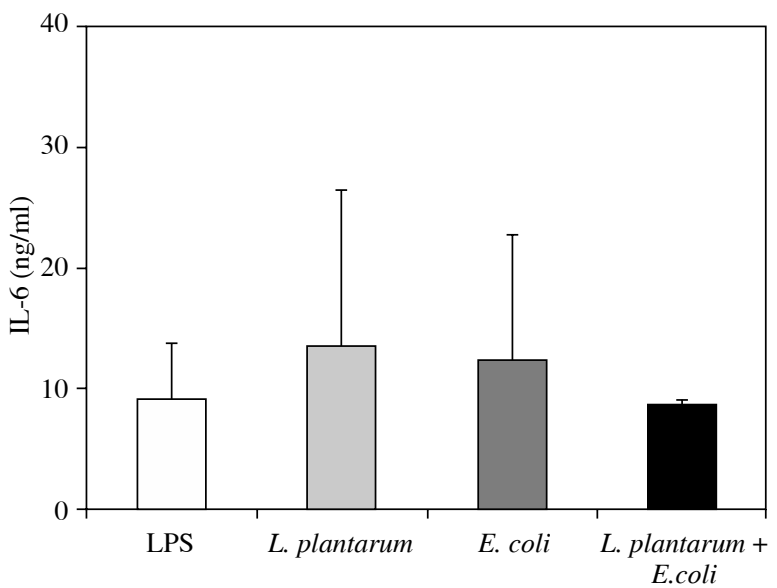

Fig. 1. The release of cytokines and NO from in vitro stimulated peritoneal exudate cells. The cells (>90\% macrophages) were stimulated in vitro with LPS $(100 \mathrm{ng} / \mathrm{ml})$, L. plantarum KL30B (live; $\left.1 \times 10^{7} \mathrm{CFU} / \mathrm{ml}\right)$, E. coli $3 \mathrm{~A} 1$ (killed; $1 \times 10^{7}$ $\mathrm{CFU} / \mathrm{ml}$ ) and L. plantarum KL30B (live) + E. coli $3 \mathrm{~A} 1$ (killed) $\left(1 \times 10^{7} \mathrm{CFU} / \mathrm{ml}\right.$ each). The release into the cell culture supernatant: A) NO, B) IL-10, C) TNF- $\alpha$ and D) IL-6 is shown as a mean ( \pm SD) of three independent experiments. $* p<0.05$ L. plantarum KL30B (live) vs. E. coli 3A1 (killed)

lesions ( 2 out of 7), and this may be interpreted as early stage of transformation from acute to chronic type of inflammation with the more severe mucosa damage and the presence of ulceration and granulation tissue. In this group MPO activity was markedly lower than in the L. plantarum KL30B group but it was not statistically significant (Table 3).

Importantly, in our experimental models, acute colitis defined by a histological evaluation strongly correlated with a high level of MPO activity, the marker of neutrophils (Fig. 5).

\section{Discussion}

In the previous studies we have shown that probiotics (lactobacilli) as well as pathogenic bacteria (E. coli) may exert either pro- or anti-inflammatory properties in a strain-specific manner [3, 19]. This observation is in agreement with a number of similar studies performed in vitro [21]. However, neither pro- nor anti-inflammatory activity of the bacterial strain tested in vitro is univocally correlated with either attenuation or exacerbation of inflammation in vivo, including ulcerative colitis. Namely, in severe systemic infection/inflammation a massive production of pro-inflammatory cytokines (e.g. TNF- $\alpha$ ) contributes to organ failure. By contrast, in experimental models of locally induced inflammation (peritonitis, colitis), the strong activity of TNF- $\alpha$ has been found to be important for effective antibacterial host defence [22]. On the other hand, in several animal studies, IL-10, a prototypic anti-inflammatory cytokine, served both a protective and pathogenic role in various bacterial infections associated with acute inflamma- 
tion [23]. Therefore, more detailed studies are necessary to compare various biological effects of potentially probiotic and pathogenic bacterial strains on the development and progression in animal models of inflammation.

In the present study we have compared the effect of selected strains of E. coli and L. plantarum on the development and propagation of acute inflammation. For this purpose, two human microbiotas, L. plantarum KL30B and E. coli 3A1, were isolated from healthy and inflamed colon, respectively. The primary criterion for such selection was the use of potentially probiotic, catalase positive Lactobacillus strain and comparing its properties with those of E. coli, the most common bacteria of gut microbiome with dual role in the pathogenesis of ulcerative colitis (IBD) $[8,24]$. The study was performed using three experimen-
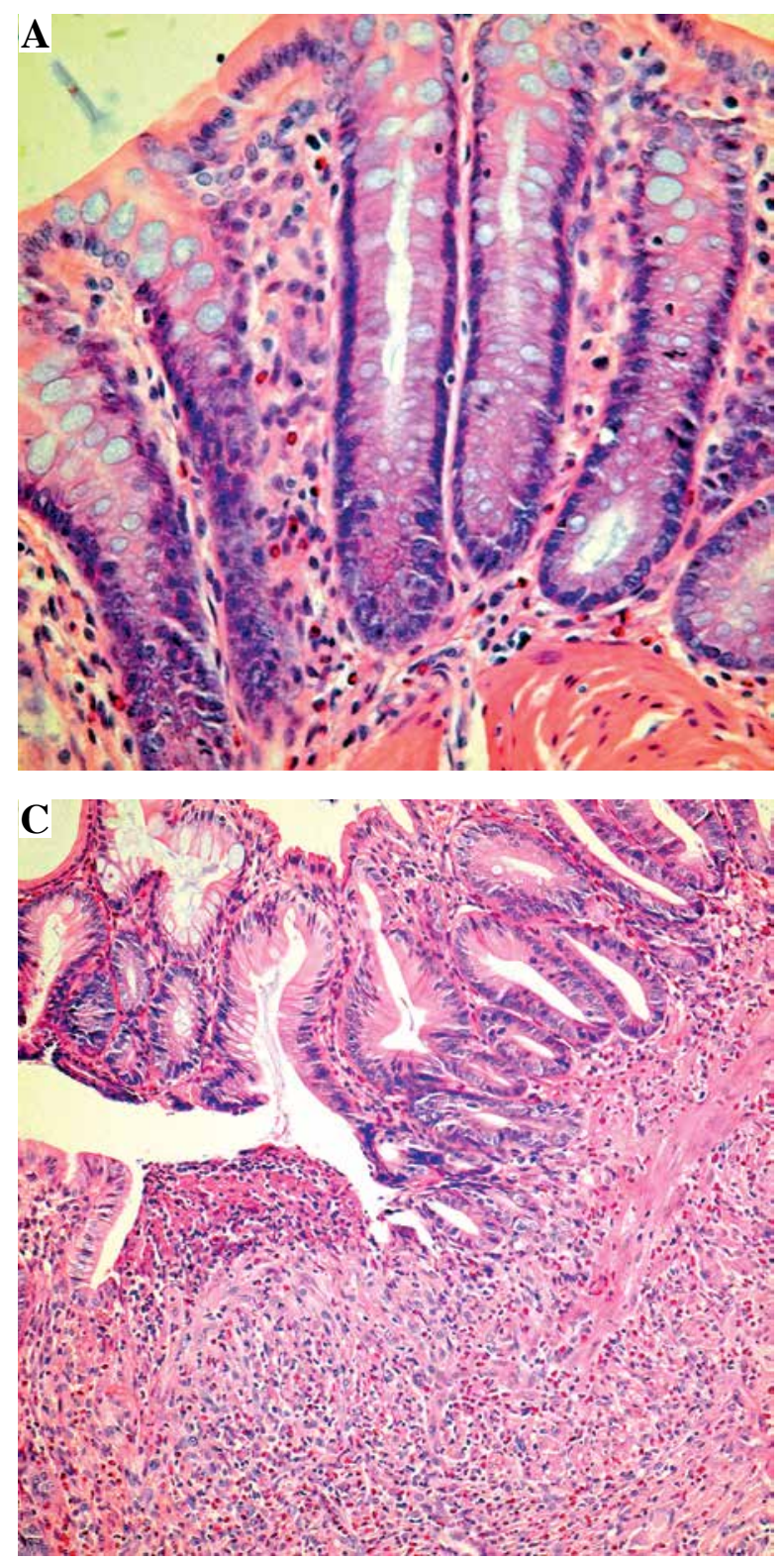

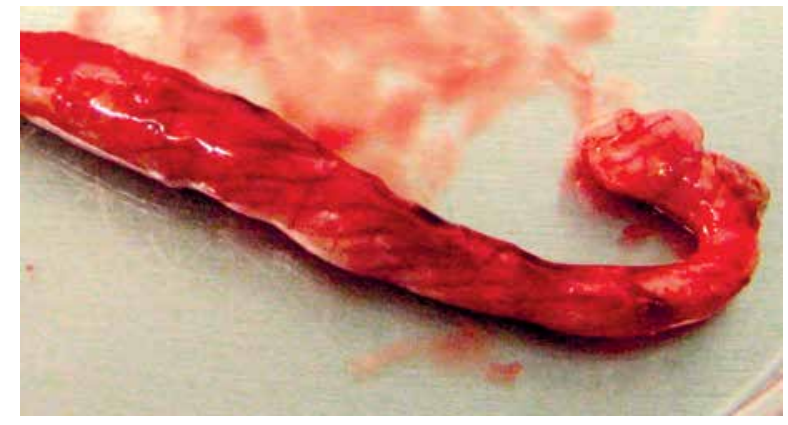

Fig. 2. The selected inflamed colon showing severe hyperaemia in TNBS-induced colitis

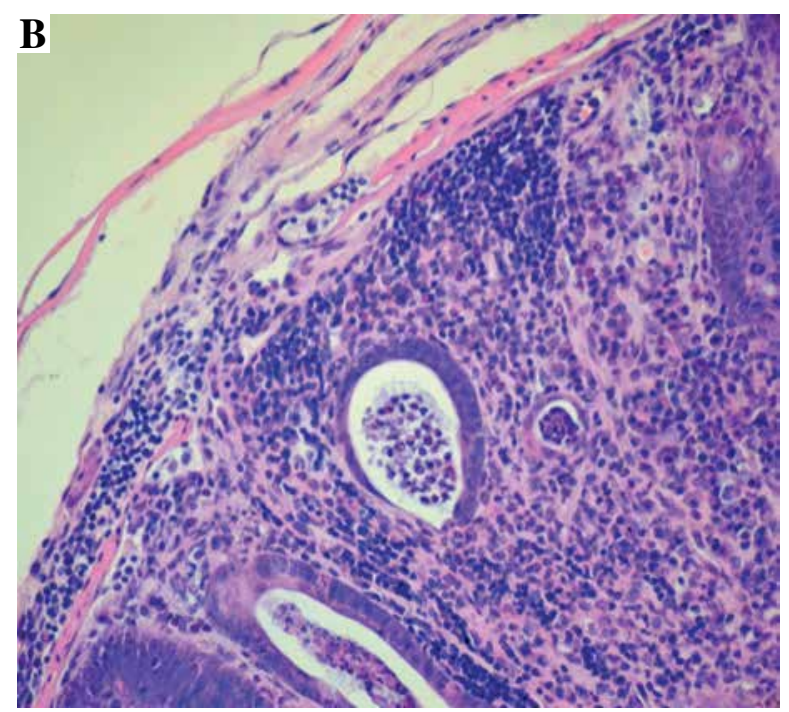

Fig. 3. Representative histological changes in rat colons stained with hematoxillin and eosin. A) A mild inflammatory infiltrate in the lamina propria; acute lesion; original magnification $400 \times$. B) Extensive inflammatory infiltrate (neutrophils) in the lamina propria and formation of microabscesses in a crypt. The epithelial cells show reduced mucus production, increased nuclear-cytoplasmic ratio and nuclear hyperchromasia ('regenerative atypia'); original magnification 400×. C) Mucosal ulceration, extensive inflammatory infiltrate, tissue granulation and the beginning of fibrosis. The inflammatory infiltrate contains neutrophils featuring acute inflammation. The presence of tissue granulation and healing indicates chronic component; original magnification $200 \times$ 
Table 3. The effect of $L$. plantarum KL30B and E. coli 3A1 administration on intestinal inflammation in TNBS-induced experimental colitis in rats

\begin{tabular}{|c|c|c|c|c|}
\hline Group $[n]$ & Incidence $[\%]$ & Histology score ${ }^{b}$ & Type of colitis $[n]$ & $\begin{array}{c}\text { MPO } \\
{\left[\mathrm{U} \times 10^{-3} / \mathrm{mg} \text { protein }\right]^{\mathrm{a}}}\end{array}$ \\
\hline TNBS control [7] & 60 & $<1$ & mild colitis [4] & $77 \pm 9^{* \#}$ \\
\hline TNBS + E. coli 3A1 (live) [5] & 60 & $3.00 \pm 0.00$ & chronic [3] & $98 \pm 22 * \#$ \\
\hline TNBS + E. coli 3A1 (killed) [5] & 60 & $2.67 \pm 0.33$ & $\begin{array}{c}\text { acute [1] } \\
\text { chronic [3] }\end{array}$ & $255 \pm 104^{* * \# \#}$ \\
\hline TNBS + L. plantarum KL30B (live) [8] & 62,5 & $2.00 \pm 0.28$ & acute [5] & $1268 \pm 351$ \\
\hline $\begin{array}{l}\text { TNBS + L. plantarum KL30B (live) }+ \text { E. coli } \\
\text { 3A1 (killed) [7] }\end{array}$ & 75 & $2.40 \pm 0.24$ & $\begin{array}{l}\text { acute }+ \text { chronic }[2] \\
\text { acute }[3]\end{array}$ & $392 \pm 46$ \\
\hline
\end{tabular}

A

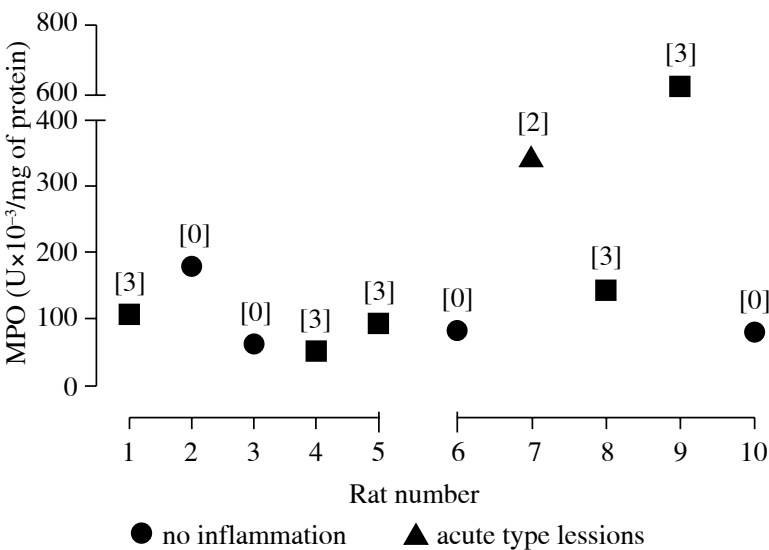

no inflammation $\quad \mathbf{A}$ acute type lessions

chronic type lessions

\section{B}

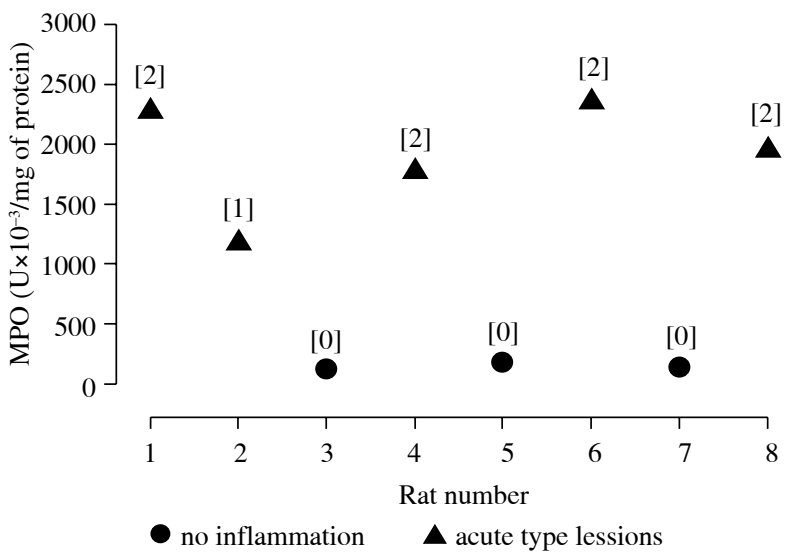

Fig. 4. MPO activity levels in mucosa fragments isolated from colons of rats treated with: A) E. coli 3A1 (live bacteria: rats no. 1-5; killed bacteria: rats no. 6-10) and B) live L. plantarum KL30B (rats 1-8). The histological grading score (see Methods) is shown in brackets [0-3]. $p<0.0001$ MPO activity levels in inflamed colons E. coli 3A1 vs. L. plantarum KL30B treated rats

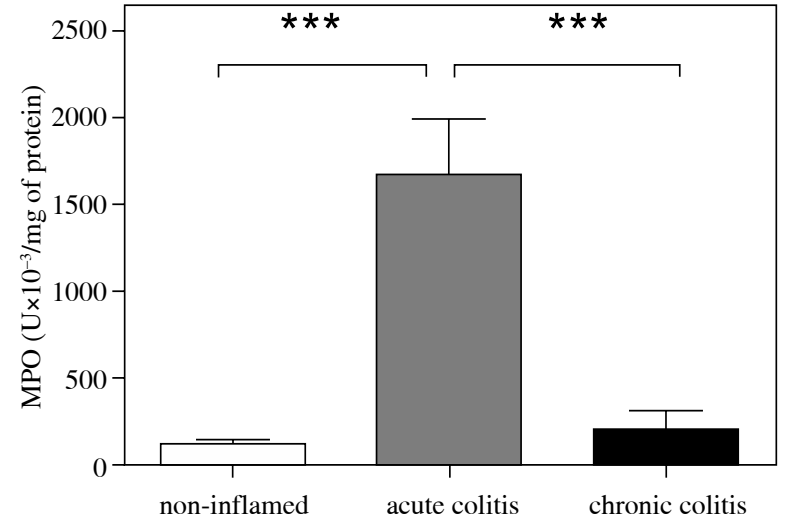

Fig. 5. MPO activity levels in mucosa fragmets of rat colons with acute and chronic colitis. $* * * p<0.0001$ acute colitis vs. non-inflamed colons and acute colitis versus chronic colitis tal models: 1) acute peritonitis in mice; 2) TNBS-induced acute colitis in rats; 3 ) in vitro activation of murine exudate peritoneal macrophages to investigate the production of inflammatory mediators.

Acute bacterial peritonitis is an acute inflammation characterized by the presence of bacteria in normally sterile peritoneal cavity, and is associated with a high mortality rate. In humans, E. coli is found in more than $60 \%$ cases. In mice models of peritonitis even low number of live $E$. coli $\left(\sim 10^{5}\right.$ bacteria/mouse) induces severe peritonitis with high mortality $[22,23]$. In our experimental model, dead $E$. coli $3 \mathrm{~A} 1$ ( $10^{7}$ bacteria/mouse) showed stronger capability to induce acute peritonitis than that achieved by administration of live L. plantarum KL30B bacteria. Both tested strains showed comparable chemotactic activity as measured by a number of infiltrating peritoneal exudate cells. However, significantly higher percentage of neutrophils $\left(\mathrm{Gr} 1^{+} \mathrm{F} 4 / 80^{-}\right.$cells) correlated with higher ac- 
tivity of MPO as observed in peritonitis induced by dead E. coli 3A1 than in peritonitis induced by live L. plantarum KL30B. Importantly, the pro-inflammatory properties of E. coli 3A1 were much stronger than LPS alone suggesting the presence of additional LPS-independent mechanism(s). In conclusion, the results presented above indicate that both tested bacterial strains may induce acute inflammation in bacteria-free tissues, such as peritoneal cavity, with much stronger virulence of $E$. coli $3 \mathrm{~A} 1$. Moreover, macrophages collected from acute peritonitis and stimulated in vitro with $L$. plantarum KL30B produce different pattern of inflammatory mediators than those stimulated with E. coli 3A1. Namely, in our experimental set-up, E. coli induced massive production of $\mathrm{NO}$, markedly higher than L. plantarum KL30B and LPS alone. Nitric oxide is toxic at high concentrations and is involved in damage of inflamed tissue $[25,26]$. It may explain the detrimental role of E. coli pathogenic strains in local inflammation [5]. Unexpectedly, E. coli was also a stronger inducer of IL-10 than L. plantarum. It may suggest its anti-inflammatory potential. However, as mentioned above, a massive local production of IL-10 at early stages of acute inflammation may also result in inhibition of TNF- $\alpha$ and may be responsible for ineffective elimination of infectious agents [27]. Thus, from this point of view the observed profile of mediators induced by L. plantarum KL30B (TNF- $\alpha^{\text {high }}$, IL- $\left.10^{\text {low }}, \mathrm{NO}^{\text {moderate }}\right)$ seems to correspond with a physiological response of innate immunity during bacterial infections [12, 24].

The next issue we addressed was whether $L$. plantarum KL30B and E. coli $3 \mathrm{~A} 1$ showing different immunoregulatory potential in the development of peritonitis would also differentially affect the development, propagation or perpetuation of acute colitis in rats. We used TNBS-induced colitis terminated at day 12, after 2 cycles of TNBS administration. Our experimental model represents the model of acute colitis corresponding to early stages of ulcerative colitis. Other reports have demonstrated that acute colitis may turn into chronic colitis after 3-4 cycles of TNBS administration [28].

The mechanism of TNBS-induced colitis is not fully explained. Proposed mechanisms by which TNBS induces colitis may be: toxic effects on the epithelium followed by destruction of mucosal barrier and increased exposure to luminal bacteria, both endogenous microbiota and exogenous bacteria applied orally during probiotic therapies [3, 13, 29, 30]. However, gnotobiotic animals should be used in further studies to evaluate a direct effect of each of the administered bacterium. Nevertheless, recognition of any invading bacteria by TLRs in lamina propria of colon may induce acute inflammation characterized by a massive infiltration of neutrophils and phagocytosis of bacterial cells. Resulting inflammation has been shown to involve not only neutrophils but also macrophages and lymphocytes [8, 31]. Importantly, acute colitis, depending on various factors, may either terminate or progress to chronicity $[7,13]$.
Majority of experimental and clinical evidence coming from different laboratories suggests that neutrophils are responsible for mucosa injury and their migration across mucosal epithelia is correlated with oxidative stress in ulcerative colitis [32, 33]. For years neutrophils have been considered simple killer cells with concomitant detrimental effect on inflamed tissues including colon mucosa in ulcerative colitis [31]. However, a number of recent studies show that both inflammatory phagocytic cells (neutrophils and macrophages) as well as NO, their common toxic product, play a dual role in the development of experimental colitis [26, 34, 35].

It has been reported that neutrophils are not essential for the development of the major pathological features of colitis [36]. Moreover, only recently it has been shown that infiltrating neutrophils modulate the mucosal microenvironment to influence resolution of inflammation [37]. What would be the explanation of such opposite effects of neutrophils on initiation, resolution/progression and severity of acute inflammation? In our opinion, diverse functions of these cells are associated with different subsets of neutrophils, namely N1 and N2 cells. It is commonly accepted in cancer research that tumour environment may shape neutrophil and macrophage polarization to two distinct final populations. In general, N1 and M1 cells exert strong pro-inflammatory and defence properties while N2 and M2 cells (TAN - tumour associated neutrophils, TAM - tumour associated macrophages) are associated with propagation of tumours $[38,39]$. As different strains of probiotic bacteria stimulate different sets of mediators released by inflammatory cells, it is tempting to speculate that bacteria can also polarise these cells (N1 vs. N2). This hypothesis is supported by the demonstration of polarization of M1 to M2-like macrophages by L. plantarum CLP-0611 [40].

Therefore, massive infiltration with neutrophils at a site of inflammation of unknown subtype (N1 vs. N2) after administration of bacteria cannot be univocally interpreted as a detrimental effect. The decisive is the final effect, either resolution of acute inflammation or progression to chronicity. The idea of probiotic therapy in IBD is to ameliorate colitis. For this purpose a number of various lactobacilli strains have been used with positive effects $[11,12,30]$. However, some immunostimulatory probiotic strains of lactobacilli amplified progression of colitis [41]. The present data clearly indicate that oral administration of L. plantarum KL30B induced massive infiltration of neutrophils correlated with high activity of MPO in inflamed mucosa in TNBS-induced acute colitis in rats. It indicates that L. plantarum KL30B has strong pro-inflammatory properties, stronger than $E$. coli $3 \mathrm{~A} 1$, as confirmed by a histological evaluation and analysis of in vitro stimulation of TNF- $\alpha$ release by macrophages. On the contrary, E. coli 3A1, but not L. plantarum KL30B, progressed TNBS-acute colitis to chronicity and caused the severe mucosal damage. What would be the explanation for high 
levels of MPO activity and mild mucosal lesions found in rats treated with L. plantarum KL30B? On one hand, MPO is responsible for the production of toxic hypochlorous acid $(\mathrm{HOCl})$ and may be responsible for tissue damage [42, 43]. On the other hand, bactericidal properties of $\mathrm{HOCl}$ may limit transmigration of endogenous gut bacteria and attenuate the development of colitis. Moreover, it has been shown that MPO suppresses the induction of iNOS and therefore the production of deleterious nitric oxide [44].

In conclusion, our comparative studies of biological properties of L. plantarum KL30B and E. coli $3 \mathrm{~A} 1$ show their distinct effect on the development and severity of acute peritonitis and colitis. We hypothesize that despite the strong pro-inflammatory properties of L. plantarum KL30B, its oral administration may have beneficial probiotic effect on the development and perpetuation of ulcerative colitis. However, to confirm our hypothesis concerning the protective role of neutrophil massive infiltration at the early stage of colitis, further studies with prolonged observation (up to 30 days) are necessary to explain the remote effect of L. plantarum KL30B resulting in either resolution of acute inflammation or in perpetuation of chronic colitis. Moreover, it is important to explain the impact of oral administration of probiotics on gut bacteria colonization and their transmigration across inflamed mucosal barrier.

Finally, we would like to stress that the final effect of bacterial therapies depends not only on the strain specific immunoregulatory properties but also on the route of administration and the fate of bacteria after homing in host tissues. Namely, the same bacterial strain may induce acute inflammatory response, may form biofilm in sterile tissues leading to chronic inflammatory response or it may crosstalk with host microbiome to attenuate ongoing inflammatory response. Therefore, it is important to remember that the response of innate immunity to bacteria administration/invasion may result in the induction of either acute or chronic inflammation or immune tolerance.

\section{Acknowledgments}

This study was supported by grants N N401 144638, N N401 042438 and 2013/09/B/NZ6/00849 from the Polish Ministry of Research and Higher Education and Jagiellonian University Medical College grant K/ZDS/004603 and K/DSC/002109.

The authors declare no conflict of interest.

\section{References}

1. Sepehri S, Kotlowski R, Bernstein CN, Krause DO (2007): Microbial diversity of inflamed and non-inflamed gut biopsy tissues in inflammatory bowel disease. Inflamm Bowel Dis 13: 675-683.
2. Campieri M, Gionchetti P (2001): Bacteria as the cause of ulcerative colitis. Gut 48: 132-135.

3. Strus M, Uhlig H, Powrie F, et al. (2005): A role of bacteria in inflammatory bowel disease evoked in animal models. Gastroenterol Pol 12: 15-20.

4. Baumgart M, Dogan B, Rishniw M, et al. (2007): Culture independent analysis of ileal mucosa reveals a selective increase in invasive Escherichia coli of novel phylogeny relative to depletion of Clostridiales in Crohn's disease involving the ileum. ISME J 1: 403-418.

5. Nataro JP, Kaper JB (1998): Diarrheagenic Escherichia coli. Clin Microbiol Rev 11: 142-201.

6. Burke DA, Axon ATR (1987): Ulcerative colitis and Escherichia coli with adhesive properties. J Clin Pathol 40: 782-786.

7. Pilarczyk-Zurek M, Chmielarczyk A, Gosiewski T, et al. (2013): Possible role of Escherichia coli in propagation and perpetuation of chronic inflammation in ulcerative colitis. BMC Gastroenterol 13: 61.

8. Sartor RB. Microbial agents in the pathogenesis, differential diagnosis, and complications of inflammatory bowel disease. In: Blaser MJ, Smith PD, Ravdin JI (eds.). Infections of the gastrointestinal tract. Raven Press, Ltd., New York 2002; 435-458.

9. Rembacken BJ, Snelling AM, Hawkey PM, et al. (1999): Non-pathogenic Escherichia coli versus mesalazine for the treatment of ulcerative colitis: a randomised trial. Lancet 354: 635-639.

10. Schultz M, Strauch UG, Linde HJ, et al. (2004): Preventive effects of Escherichia coli strain Nissle 1917 on acute and chronic intestinal inflammation in two different murine models of colitis. Clin Diagn Lab Immunol 11: 372-378.

11. Peran L, Camuesco D, Comalada M, et al. (2005): Preventative effects of a probiotic, Lactobacillus salivarius ssp. salivarius, in the TNBS model of rat colitis. World J Gastroenterol 11: 5185-5192.

12. Peran L, Sierra S, Comalada M, et al. (2007): A comparative study of the preventative effects exerted by two probiotics, Lactobacillus reuteri and Lactobacillus fermentum, in the trinitrobenzenesulfonic acid model of rat colitis. Br J Nutr 97: 96-103.

13. Melgar S, Karlsson A, Michaëlsson E (2005): Acute colitis induced by dextran sulfate sodium progresses to chronicity in $\mathrm{C} 57 \mathrm{BL} / 6$ but not in BALB/c mice: correlation between symptoms and inflammation. Am J Physiol Gastrointest Liver Physiol 288: G1328-G1338.

14. Camuesco D, Peran L, Comalada M, et al. (2005): Preventative effects of lactulose in the trinitrobenzenesulphonic acid model of rat colitis. Inflamm Bowel Dis 11: 265-271.

15. Wengrower D, Zanninelli G, Latella G, et al. (2012): Losartan reduces trinitrobenzene sulphonic acid- induced colorectal fibrosis in rats. Can J Gastroenterol 26: 33-39.

16. Yukitake H, Kimura H, Suzuki H, et al. (2011): BTZO-15, and ARE-activator, ameliorates DSS- and TNBS-induced colitis in rats. PLoS One 6: e23256.

17. Marzinzig M, Nussler AK, Stadler J, et al. (1997): Improved methods to measure end products of nitric oxide in biological fluids: nitrite, nitrate, and S-nitrosothiols. Nitric Oxide 1: 177-189.

18. Bradley RP, Christensen RD, Rothstein G (1982): Cellular and extracellular myeloperoxidase in pyogenic inflammation. Blood 60: 618-622.

19. Marcinkiewicz J, Ciszek M, Bobek M, et al. (2007): Differential inflammatory mediator response in vitro from murine 
macrophages to lactobacilli and pathogenic intestinal bacteria. Int J Exp Pathol 88: 155-164.

20. Larson SJ, Collins SM, Weingarten HP (1996): Dissociation of temperature changes and anorexia after experimental colitis and LPS administration in rats. Am J Physiol 271: R967-972.

21. Peńa JA, Li SY, Wilson PH, et al. (2004): Genotypic and phenotypic studies of murine intestinal lactobacilli: species differences in mice with and without colitis. Appl Environ Microbiol 70: 558-568.

22. Knapp S, de Vos AF, Florquin S, et al. (2003): Lipopolysaccharide binding protein is an essential component of the innate immune response to Escherichia coli peritonitis in mice. Infect Immun 71: 6747-6753.

23. Sewnath ME, Olszyna DP, Birjmohun R, et al. (2001): IL-10-deficient mice demonstrate multiple organ failure and increased mortality during Escherichia coli peritonitis despite an accelerated bacterial clearance. J Immunol 166: 6323-6331.

24. Nakanishi Y, Sato T, Ohteki T (2015): Commensal Gram-positive bacteria initiates colitis by inducing monocyte/macrophage mobilization. Mucosal Immunol 8: 152-160.

25. McCafferty DM, Miampamba M, Sihota E, et al. (1999): Role of inducible nitric oxide synthase in trinitrobenzene sulphonic acid induced colitis in mice. Gut 45: 864-873.

26. Cross RK, Wilson KT (2003): Nitric oxide in inflammatory bowel disease. Inflamm Bowel Dis 9: 179-189.

27. Li B, Alli R, Vogel P, Geiger TL (2014): IL-10 modulates DSS-induced colitis through a macrophage-ROS-NO axis. Mucosal Immunol 7: 869-878.

28. Zhu MY, Lu YM, Ou YX, et al. (2012): Dynamic progress of 2, 4, 6-trinitrobenzene sulfonic acid induced chronic colitis and fibrosis in rat model. J Dig Dis 13: 421-429.

29. Rath HC, Schultz M, Freitag R, et al. (2001): Different subsets of enteric bacteria induce and perpetuate experimental colitis in rats and mice. Infect Immun 69: 2277-2285.

30. Satish Kumar CS, Kondal Reddy K, Reddy AG, et al. (2015): Protective effect of Lactobacillus plantarum 21, a probiotic on trinitrobenzenesulfonic acid-induced ulcerative colitis in rats. Int Immunopharmacol 25: 504-510.

31. Sartor RB (1997): Pathogenesis and immune mechanisms of chronic inflammatory bowel diseases. Am J Gastroenterol 92: $5 \mathrm{~S}-11 \mathrm{~S}$.

32. Naito Y, Takagi T, Yoshikawa T (2007): Neutrophil-dependent oxidative stress in ulcerative colitis. J Clin Biochem Nutr 41: $18-26$

33. Yasukawa K, Tokuda H, Tun X, et al. (2012): The detrimental effect of nitric oxide on tissue is associated with inflammatory events in the vascular endothelium and neutrophils in mice with dextran sodium sulfate- induced colitis. Free Radic Res 46: 1427-1436.

34. Rumi G, Tsubouchi R, Nishio H, et al. (2004): Dual role of endogenous nitric oxide in development of dextran sodium sulfate-induced colitis in rats. J Physiol Pharmacol 55: 823836.

35. Tun X, Yasukawa K, Yamada K (2014): Involvement of nitric oxide with activation of Toll-like receptor 4 signaling in mice with dextran sodium sulfate- induced colitis. Free Radic Biol Med 74: 108-117.

36. Buell MG, Berin MC (1994): Neutrophil-independence of the initiation of colonic injury. Comparison of results from three models of experimental colitis in the rat. Dig Dis Sci 39: 2575-2588.

37. Campbell EL, Bruyninckx WJ, Kelly CJ, et al. (2014): Transmigrating neutrophils shape the mucosal microenvironment through localized oxygen depletion to influence resolution of inflammation. Immunity 40: 66-77.

38. Fridlender ZG, Albelda SM (2012): Tumor-associated neutrophils: friend or foe? Carcinogenesis 33: 949-955.

39. Piccard H, Muschel RJ, Opdenakker G (2012): On the dual roles and polarized phenotypes of neutrophils in tumor development and progression. Crit Rev Oncol Hematol 82: 296309.

40. Jang SE, Han MJ, Kim SY, Kim DH (2014): Lactobacillus plantarum CLP- 0611 ameliorates colitis in mice by polarizing M1 to M2- like macrophages. Int Immunopharmacol 21: 186-192.

41. Mileti E, Matteoli G, Iliev ID, Rescigno M (2009): Comparison of the immunomodulatory properties of three probiotic strains of Lactobacilli using complex culture systems: prediction for in vivo efficacy. PLoS One 4: e7056.

42. Klebanoff SJ (2005): Myeloperoxidase: friend and foe. J Leukoc Biol 77: 598-625.

43. Prokopowicz Z, Marcinkiewicz J, Katz DR, Chain BM (2012): Neutrophil myeloperoxidase: soldier and statesman. Arch Immunol Ther Exp 60: 43-54.

44. Kumar AP, Ryan C, Cordy V, Reynolds WF (2005): Inducible nitric oxide synthase expression is inhibited by myeloperoxidase. Nitric Oxide 13: 42-53. 